\title{
CONVOLUTION INEQUALITIES ON FINITE CYCLIC GROUPS AND THE PSEUDOMEASURE NORM
}

\author{
DAVID L. RITTER
}

\begin{abstract}
A characterization of the probability measures that define hypercontractive convolution operators on finite cyclic groups has been given in terms of the pseudomeasure norm. Here the pseudomeasure norm is shown to be a poor quantitative predictor of hypercontractiveness in an asymptotic sense.
\end{abstract}

For $K \geqslant 2$, let $G_{K}$ denote the additive cyclic group $Z / K Z=\{0,1, \ldots, K-1\}$, and for $p \geqslant 1$, let $L^{p}\left(G_{K}\right)$ denote the usual Banach space of $p$ th power summable functions on $G_{K}$ with norm $\|\cdot\|_{p . K}$ defined in terms of the Haar measure $m_{K}$ that assigns mass $1 / K$ to each point. Let $\Gamma_{K}$ denote the group dual to $G_{K}$ supplied with ordinary counting measure so that the Plancherel theorem is valid for the pair of groups $G_{K}, \Gamma_{K}$. Finally, let $P\left(G_{K}\right)$ denote the set of probability measures on $G_{K}$.

Fix $p>1$ and $\mu$ in $P\left(G_{K}\right)$. If there is a $q>p$ such that

$$
\|\mu * f\|_{q, K} \leqslant\|f\|_{p, K}, \quad f \in L^{p}\left(G_{K}\right),
$$

then we say $\mu$ is a hypercontraction.

The interest in hypercontractive measures on finite cyclic groups originated in the study of convolution properties of particular measures defined on the circle group, the Cantor group, and the real line; see [1-5, and 8]. Currently, however, the dependence of $p$ and $q$ in (1) on the measure $\mu$ is really completely understood on only one group: $G_{2}=Z / 2 Z$. In this case, Bonami [3], Beckner [1], and Weissler [8] have shown that

$$
(q-1)|\hat{\mu}(1)|^{2} \leqslant p-1
$$

is necessary and sufficient for (1) to be true, where $\hat{\mu}$ is the Fourier-Stieljes transform of $\mu$.

Although the determination of the precise relationship between $p$ and $q$ in (1) for an arbitrary measure $\mu$ is difficult, a characterization of hypercontractive probability measures in terms of the pseudomeasure norm is known $[\mathbf{2 , 6 ]}$ :

THEOREM 1. (a) $\mu$ in $P\left(G_{K}\right)$ satisfies (1) for some $q>p$ if, and only if,

$$
\left\|\left(m_{K}-\mu\right)^{\hat{n}}\right\|_{\infty}<1 .
$$

Received by the editors March 31, 1983.

1980 Mathematics Subject Classification. Primary 43A22, 43A15.

"1984 American Mathematical Society $0002-9939 / 84 \$ 1.00+\$ .25$ per page 
(b) In addition, if $p>1$ and $0<\xi<1$, then there is a $q>p$ such that (1) is true for $\mu$ in $P\left(G_{K}\right)$ whenever

$$
\left\|\left(m_{K}-\mu\right)^{\wedge}\right\|_{\infty} \leqslant \xi .
$$

If we let $q(K, \xi, p)$ denote the supremum of the $q>p$ such that (4) implies (1) on $G_{K}$, then our main result is the theorem that follows.

THEOREM 2. If $p>1$ and $0<\xi<1$, then $\lim _{K \rightarrow x} q(K, \xi, p)=p$.

To prove Theorem 2, we work on the circle group $T=R / Z$ which we realize as the interval $[0,1)$. Consequently, for $K \geqslant 2$, we take $G_{K}=\{j / K: j=0,1, \ldots, K-$ $1\}$. Then it turns out that our theorem is a consequence of the following two lemmas.

LEMMA 1. Let $\mu$ be a Borel measure on the circle group, and let $p \geqslant 1$. If there is a $q>p$ and $C>0$ such that

$$
\|\mu * f\|_{q} \leqslant C\|f\|_{p}
$$

for each $f$ in $L^{p}(T)$, then $\mu$ is a continuous measure.

Lemma 2. For each $K \geqslant 2$, let $\mu_{K}$ be a probability measure on $T$ with support in $G_{K}$. Suppose there is a $p \geqslant 1$ and $q>p$ such that (1) is true for $\mu_{K}$ for infinitely many $K$. If $\mu$ is a Borel measure on $T$ such that $\mu_{K} \rightarrow \mu$ weak* as $K \rightarrow \infty$, then $\mu$ satisfies (5) for $f$ in $L^{p}(T)$ with $C=1$.

Proof of LEMMA 1. By a standard interpolation involving Young's inequality for measures and the Riesz-Thorin interpolation theorem, we may suppose $q=2$ and $p<q$ in (5). By convolving $\mu$ with the Dirichlet kernel $D_{K}(x)=\sum_{|j| \leqslant K} \operatorname{Exp}(2 \pi i j x)$, using the fact that $\left\|D_{K}\right\|_{p} \sim K^{1 / p^{\prime}}$, where $(1 / p)+\left(1 / p^{\prime}\right)=1$, and applying (5), we obtain

$$
\sum_{|j| \leqslant K}|\hat{\mu}(j)|^{2} \leqslant C_{0} K^{2 / p^{\prime}} \leqslant C_{0}(2 K+1)^{2 / p^{\prime}},
$$

where $C_{0}$ is independent of $K$. Since $p^{\prime}>2$, the lemma follows from Wiener's theorem on the average value of $|\hat{\mu}|^{2}$ once we divide both sides of the inequality above by $2 K+1$ and let $K \rightarrow \infty$.

Proof of Lemma 2. It suffices to show (5) holds with $f$ a continuous complex-valued function on the circle. Thus, let $f$ be continuous, and for $K \geqslant 2$, let $f_{K}$ be the restriction of $f$ to $G_{K}$, and let $I(K, j)=[j / K,(j+1) / K)$ for $j=0, \ldots, K-1$. The sequence of functions $\left\{\mu_{K} * f\right\}$ is uniformly equicontinuous, and since $\mu_{K} \rightarrow \mu$ weak*, we have $\mu_{K} * f \rightarrow \mu * f$ pointwise. Consequently,

$$
\left\|\mu_{K} * f-\mu * f\right\|_{\infty} \rightarrow 0 \text { as } K \rightarrow \infty \text {. }
$$

Next, from

$$
\left\|\mu_{K} * f\right\|_{q}^{q}-\left\|\mu_{K} * f_{K}\right\|_{q, K}^{q}=\sum_{j=0}^{K-1} \int_{I(K, j)}\left|\mu_{K} * f(x)\right|^{q}-\left|\mu_{K} * f_{K}(j / K)\right|^{q} d x
$$


and

$$
\mu_{K} * f(x)-\mu_{K} * f_{K}\left(\frac{j}{K}\right)=\sum_{i=0}^{K-1}\left[f\left(x-\frac{i}{K}\right)-f\left(\left(\frac{j-i}{K}\right)\right)\right] \mu_{K}\left(\frac{i}{K}\right)
$$

it is easy to see

$$
\left|\left\|\mu_{K} * f\right\|_{q}-\left\|\mu_{K} * f_{K}\right\|_{q, K}\right| \rightarrow 0 \quad \text { as } K \rightarrow \infty .
$$

Similarly, we have

$$
\left|\|f\|_{p}-\left\|f_{K}\right\|_{p . K}\right| \rightarrow 0 \quad \text { as } K \rightarrow \infty .
$$

To see how (5) follows from (1) and (6)-(8), let $\varepsilon>0$. Then for some $K$ sufficiently large we have

$$
\begin{aligned}
\|\mu * f\|_{q} & \leqslant\left\|\mu * f-\mu_{K} * f\right\|_{q}+\left\|\mu_{K} * f\right\|_{q} \\
& \leqslant\left\|\mu * f-\mu_{K} * f\right\|_{\infty}+\left\|\mu_{K} * f_{K}\right\|_{q, K}+\varepsilon \\
& \leqslant\left\|f_{K}\right\|_{p, K}+2 \varepsilon \leqslant\|f\|_{p}+3 \varepsilon,
\end{aligned}
$$

and the lemma is proved.

We now prove Theorem 2.

Proof of Theorem 2. Fix $p>1$ and $0<\xi<1$. By Theorem $1(\mathrm{~b}), q(K, \xi, p)>p$ for each $K \geqslant 2$. Therefore, it suffices to show

$$
\lim \sup q(K, \xi, p)=p \text {. }
$$

We obtain (9) by an indirect argument. Suppose (9) is false, and fix $q$ with

$$
\limsup q(K, \xi, p)>q>p .
$$

Then for infinitely many $K$, on $G_{K}$ we have (4) implying (1) with $\xi, p$, and $q$ as above. For each $K \geqslant 2$, define $\mu_{K}$ on $G_{K}$ by $\mu_{K}=\xi \delta_{0}+(1-\xi) m_{K}$, where $\delta_{0}$ is the point mass at 0 on $G_{K}$. Then for $j$ in $\Gamma_{K},\left(m_{K}-\mu_{K}\right) \hat{(j)}=-\xi$ when $j \neq 0$, and $\left(m_{K}-\mu_{K}\right) \hat{(0)}=0$. Therefore $\mu_{K}$ satisfies (4). Consequently (1) is true for infinitely many $\mu_{K}$. From Lemma $2, \mu=\xi \delta_{0}+(1-\xi) d x$, the weak* limit of the $\mu_{K}$ 's, satisfies (5), and hence by Lemma 1 is continuous, a patent absurdity.

REMARKS. (a) The method of proof of Theorem 1 found in [2] is superior to that of [6] because the method used in [2] provides an estimate of $q(K, \xi, p)$. By using the elementary inequality $\|f\|_{4, K} \leqslant K^{1 / 4}\|f\|_{2, K}$ and by following the line of the proof of Lemma 2 of [2], one can readily show

$$
q(K, \xi, 2) \geqslant \frac{4 \ln \left(\xi^{-1}\right)-2 \ln \left(3 K^{1 / 2}\right)}{\ln \left(\xi^{-1}\right)-\ln \left(3 K^{1 / 2}\right)} .
$$

Consequently, an application of the Riesz-Thorin interpolation theorem and Young's inequality for measures yields a lower bound for $q(K, \xi, p)$ whenever $1<p<\infty$.

(b) Each measure $\mu_{K}$ appearing in the proof of Theorem 2 is obviously extremal with respect to $p$ and $\xi$ satisfying $q(K, \xi, p)=2$. It would be of interest to know whether it is extremal for every $p$ and $\xi$. 
(c) An earlier version of this paper claimed, erroneously, to have settled a conjecture due to Beckner, Janson, and Jerison found in [2]. To our knowledge that conjecture remains open.

\section{REFERENCES}

1. W. Beckner, Inequalities in Fourier analysis, Ann. of Math. (2) 102 (1975), 159-182.

2. W. Beckner, S. Janson and D. Jerison, Convolution inequalities on the circle, Conference on Harmonic Analysis in Honor of Antoni Zygmund, (W. Beckner et al., eds.), Wadsworth International Group, Belmont, Calif., 1983, pp. 32-43.

3. A. Bonami, Etude des coefficients de Fourier des fonctions de $L^{p}(G)$, Ann. Inst. Fourier (Grenoble) 20 (1970), 335-402.

4. D. Oberlin, A convolution property of the Cantor-Lebesgue measure, Colloq. Math. (to appear).

5. D. Ritter, Some singular measures on the circle which improve $L^{p}$ spaces, Colloq. Math. (to appear).

6. A A convolution theorem for probability measures on finite groups, Illinois J. Math. (to appear).

7. E. M. Stein, Harmonic analysis on $R^{\prime \prime}$, Studies in Harmonic Analysis, MAA Studies in Math., Vol. 13, Mathematical Association of America, Washington, D.C., 1976, pp. 97-135.

8. F. B. Weissler, Two-point inequalities, the Hermite semigroup, and the Gauss-Weierstrass semigroup, J. Funct. Anal. 32 (1970), 102-121.

Department of Mathematical Sciences, Florida International University, Miami, Florida 33199 